assumptions about technological development. Dynamic modelling is just as vulnerable to implicit error in this regard. William E. Rees

School of Community and Regional Planning,

University of British Columbia,

6333 Memorial Road, Vancouver,

British Columbia V6T 1Z2, Canada

\section{Xenotransplantation's benefits outweigh risks}

Sir - Your News item "Diabetes trial stirs debate on safety of xenotransplants" (Nature 419,$5 ; 2002$ ), discussing our clinical trial of pancreatic islet xenotransplantation, states: "Mexico has no published guidelines on clinical trials in xenotransplantation."

This is untrue. Mexico has a general health law that regulates the conduct of all experimental investigation in human beings; it also regulates organ and tissue transplantation, and xenotransplantation, which was added recently. The protocol for our clinical trial was approved by the research, ethics and biosafety committees of our institution and of the National Autonomous University of Mexico Medical School.

Your article did not mention the existence of other published studies on porcine-human islet xenotransplantation, although these studies are admittedly small, use different methods from ours and do not show significant benefit to patients. With regard to your reporter's comment about the need to test "any proposed approach" on non-human primates, there are many examples in the fields of both transplantation and diabetes where experimental studies in humans are performed following evidence of benefit derived only from other mammalian models. Indeed, one of the problems we faced was that there is no appropriate model for auto-immune diabetes in non-human primates.

You report our critics' views that we should have undertaken preclinical studies before proceeding, but vast amounts of preclinical data on animal models already exist, both on efficacy of islet xenotransplantation and the protective effects of Sertoli cells, including our own published work. We have not published some of our work, to protect patents. But we consider the available literature sufficient to justify our trials on young diabetic patients, the population that stands to benefit most.

You report our critics as stating that our work was on patients too young to give proper informed consent. We recruited adolescent and high-school students because they had had diabetes type 1 for more than four years, so that spontaneous remission would not occur, yet there were no diabetic complications typical of adult patients. It is our strong contention that they were old enough to understand fully the potential risks and responsibilities. We had several meetings with the patients and their parents to explain in detail the procedure. After time for reflection, they signed extensive informed consent forms conforming to international requirements, for example the Helsinki declaration.

Type 1 diabetes causes the death of more than $40 \%$ of patients before they reach the age of 40 , and is a leading cause of terminal renal insufficiency and blindness, among other devastating complications. We believe that the benefits of better metabolic control, and insulin and immunosuppression independence, offered by pancreatic islet xenotransplantation far outweigh the risks.

Rafael Valdes Gonzalez

Hospital Infantil de México "Federico Gomez", Laboratorio de Xenotrasplantes, Edificio Mundet $4^{\circ}$ Piso, Calle Dr Marquez 162, Col. Doctores, CP 06720, México D.F.

\section{UK government closes its eyes to medical needs}

Sir - Readers of Nature may get the impression that funding for basic research in the United Kingdom is in good health ("Calling for entrepreneurs: London" Naturejobs 19 September, 4-5; 2002). We wish to put the record straight.

The UK government uses the periodic Research Assessment Exercise (RAE) to determine levels of funding for university departments and institutes. Following the latest RAE, funding for hospital-based clinical subjects, which underpins the core of translational research in medical schools across the United Kingdom, was severely cut. Despite having received, for the third consecutive time, the highest possible rating $\left(5^{*}\right)$, the Institute of Ophthalmology in London faces a $19.7 \%$ cut in its predicted annual budget because the government has failed to honour its pre-RAE fiscal commitments. The problem is even greater for those who received a rating of 5 and who now face a massive $38 \%$ cut.

This takes $\mathfrak{£} 970,000$ (US\$1.5 million) a year out of our operating budget. We are unable to appoint even a single technical post that we require to run newly commissioned laboratories, which puts extra stress on academic staff. The cuts also exacerbate the financial difficulties faced by our parent body, University College London, after years of underfunding.

The reason UK medical schools are having problems is self-evident, and the crisis extends across the country. In the longer term our institute, together with others in hospital-based clinical subjects, will be less able to fulfil its mission, which is to deliver the translational research that enables laboratory science to be developed into clinical trials and ultimately into benefits to patients.

The message appears to be that, with regard to translational medical research, the government is not only blind to the achievements of $5^{\star}$ departments and institutes, it has also, incomprehensibly, decided to slash the support they receive. We also suspect that the cuts are due at least in part to inept planning and muddled thinking. Institutes such as ours have to budget, recruit and develop a coherent research strategy within the constraints of government policy. It is therefore essential that the government keeps its promises. This it has not done. In the United Kingdom the phrase 'science policy' has become an oxymoron.

If, as I. B. Holland suggests (Nature 419, 248 ; 2002), underfunding is less acute here than in other European countries, then the prospects for our continent of beleaguered researchers must be bleak. Governments are often short-sighted when it comes to policy development, but in this case we are confronted by a total lack of vision. And as we are only too well aware at this institute, myopia may be corrected but blindness remains virtually untreatable.

Stephen E. Moss, Gary Rubin, John Greenwood, Adam Sillito Institute of Ophthalmology, University College London, 11-43 Bath Street, London EC1V 9EL, UK

\section{Mother knows best}

Sir - In his otherwise excellent News and Views "The grand assault" (Nature 419, 493-494; 2002), Russell F. Doolittle writes: "Eukaryotes can be loosely defined as organisms whose cells have nuclei and cytoskeletons, distinguishing them from the Bacteria and the Archaea, neither of which has introns in their coding sequences."

Bacteria and the phage that infect them do contain introns in their genomes. The first example of an intron in a bacterial system was found in the thymidylate synthase gene of bacteriophage T4 (F. K. Chu, G. F. Maley, F. Maley and M. Belfort Proc. Natl Acad. Sci. USA 81, 3049-3053; 1984) — the last author being my mother. Since then, hundreds of introns have been found in archaea, bacteria and their phage.

It has been a long time since I uttered these words, but I couldn't be more proud to say: "Mommy told me so."

\section{Gabriel M. Belfort}

Department of Biochemistry, K-building,

Boston University School of Medicine, 715 Albany

Street, Boston, Massachusetts 02118, USA 